Kong. Res. J. 2(2) : 6-10, 2015

Kongunadu Arts and Science College, Coimbatore.

\title{
GREEN SYNTHESIS OF SILVER NANOPARTICLES USING GRAVIOLA LEAF AQUEOUS EXTRACT AT ROOM TEMPERATURE
}

\author{
Ranjithkumar, R${ }^{1}$., B. Chandar Shekar ${ }^{* 2}$, C.K. Senthil Kumaran ${ }^{2}$, C. Sharmila ${ }^{3}$ and V. Simi2 \\ 1Bionano Research Center, The Nilgiris Education and Research Foundation, The Nilgiris, Coimbatore. \\ ${ }^{2}$ Department of Physics, Kongunadu College of Arts and Science, Coimbatore, Tamilnadu, India. \\ ${ }^{3}$ Department of Physics, PSGR Krishnammal College for Women, Coimbatore, Tamilnadu India. \\ *E.mail: chandar.bellan@gmail.com
}

\section{ABSTRACT}

Improvement of green route for the synthesis of silver nanoparticles with plant extracts plays a very important role in nanotechnology without any harmful chemicals. The present investigation demonstrates the synthesis of silver nanoparticles by treating silver nitrate with Graviola leaf extract at room temperature. The effect of the extract on the formation of silver nanoparticles was characterized by UV-Vis spectrum, Fourier transform infrared spectroscopy (FT-IR), X-ray diffraction (XRD) and Scanning electron microscopic (SEM) analysis. The UV-Vis spectra results show a strong resonance centered on the surface of silver nanoparticles (AgNPs) at 400-450 $\mathrm{nm}$. The Fourier transformation infrared spectroscopy spectral study demonstrates Graviola leaf aqueous extract acted as the reducing and stabilizing agent during the synthesis of silver nanoparticles. XRD and SEM studies revealed that the synthesized silver nanoparticles shows spherical in shape with average particles size around $30-70 \mathrm{~nm}$.

Keywords: Silver nanoparticles, Graviola leaf, aqueous extract.

\section{INTRODUCTION}

Nanoparticles are of great scientific interest as they effectively bridge between bulk material and atomic or molecular structure. The two principles ways of preparing nanoscale materials are "Bottom up" and "Top down" approaches. In bottom up approach, materials and devices are built from molecular compounds which assemble themselves using the principle of molecular. In top down approach, nano objects are constructed from larger entities without atomic level control, when we bring constituents of materials down to nanoscale. Nanotechnology finds wide range of applications such as medicine, information technologies, biotechnologies, energy production and storage, material technologies, manufacturing instrumentation, environmental application and security (Wang and Xia, 2014). In biological science, many applications of metal nanoparicles are being explored including labels for cells and biomolecules and cancers therapies among noble metals nanoparticles, silver nanoparticles have received considerable attention due to their attractive physiochemical properties. Silver nanoparticle reserve important role in the electronic industries and they also exhibit remarkable size effect in biological and medicine related properties (Protima et al., 2015). Basically most of the physical and chemical methods used to synthesis of nano material are too expensive and also involve the use of toxic, hazardous chemicals that are responsible for various biological risks. It is very important to develop ecofriendly processes through green route and other biological approaches using microbes, enzymes and plant materials (Ranjithkumar et al., 2013). The present study deals with synthesis of silver nanoparticles from green route using Graviola plant leaf aqueous extract at room temperature.

\section{MATERIALS AND METHODS}

\subsection{Materials}

The chemical silver nitrate $\left(\mathrm{AgNO}_{3}\right)$ was purchased from SD Fine Chemical Pvt. Ltd., Mumbai.

\subsection{Plant material}

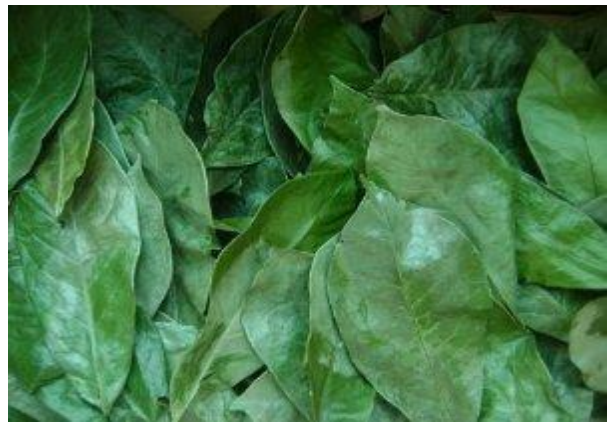

Fig.1: The leaves of Graviola collected from Kannur district, Kerala. 
The leaf of Graviola (Annona muricata) was collected from Kannur district, Kerala, India. The plant was identified Annona muricata common name Graviola (Fig. 1) at the Department of Botany, Government Arts College, Ooty, Tamilnadu, India.

\subsection{Preparations of the extract}

The Graviola plant leaf was rinsed with distilled water thoroughly to remove dirt and other attached particles. The Graviola aqueous leaf extract was prepared by taking $5 \mathrm{~g}$ of thoroughly washed and finely cut Graviola leaf in a $100 \mathrm{ml}$ Erlenmeyer flask with $50 \mathrm{ml}$ of sterile distilled water and then boiled the mixture for $5 \mathrm{~min}$. The solution was then removed from the heat source and left at room temperature. Following this step the extract was then filtered through a normal filter paper followed by Whatman filter paper No. 1 . The extract was kept in refrigerator at $4^{\circ} \mathrm{C}$ for further experiments.

\subsection{Synthesis of silver nanoparticles}

The aqueous solution of silver nitrate $\left(\mathrm{AgNO}_{3}\right)$ at concentration of $0.002 \mathrm{M}$ was prepared to synthesize silver nanoparticles from aqueous extract of Graviola leaf. $50 \mathrm{ml}$ of aqueous solution of $0.002 \mathrm{M} \mathrm{AgNO}_{3}$ was slowly added to $10 \mathrm{ml}$ of Graviola aqueous leaf extract while stirring for reduction into Ag ions. The formation of dark brown colour was observed after $1 \mathrm{~h}$ incubation at room temperature and lambda max was taken using UV-Visible spectroscopy (JASCO V-670). Then the silver nanoparticles solution was purified by repeated centrifugation at 6,000 rpm for $30 \mathrm{~min}$ to isolate $\mathrm{Ag}$ nanoparticles free from other bioorganic compounds present in the reaction medium. After centrifugation the obtained particles were washed with distilled water for 5 to $10 \mathrm{~min}$ and kept it in Hot air oven for drying at $100^{\circ} \mathrm{C}$ for $1 \mathrm{~h}$.

\subsection{Characterization techniques}

\subsubsection{UV-Visible spectroscopy}

Formation of silver particles (after $1 \mathrm{~h}$ incubation at room temperature) was confirmed by the colour change of the solution and the surface plasmon resonance band was obtained by UV-Visible spectral analysis which was done by using UV-Visible spectrophotometer (JASCO, V-670) from 300-700 $\mathrm{nm}$ at a resolution of $1 \mathrm{~nm}$.

\subsubsection{Fourier transform infrared spectrum (FTIR)}

After 1 hour incubation the silver nanoparticles were isolated by repeated centrifugation and $100^{\circ} \mathrm{C}$ for $1 \mathrm{~h}$. The obtained dried silver nanoparticles were subjected to Fourier transform infrared (FTIR) spectrum analysis in the range from 4,000 to $400 \mathrm{~cm}^{-1}$ on IR-Tracer-100 shimadzu FT-IR spectrophotometer.

\subsubsection{X-ray Diffraction spectrum}

X-ray diffraction (XRD) measurement of the green synthesized using Graviola aqueous leaf extract reduced silver particles was carried out using X'Pert Pro X-ray diffractometer (PAN analytical BV, The Netherlands) equipped with $\mathrm{Cu} \mathrm{K} \alpha$ radiation source using $\mathrm{Ni}$ as filter at a setting of $30 \mathrm{kV} / 30 \mathrm{~mA}$. All X-ray diffraction data were collected under the experimental conditions in the regular angular range.

The crystalline silver nanoparticle was calculated from the width of the XRD peaks, using a Debye-Scherer formula,

$$
\mathrm{D}=\frac{0.94 \lambda}{\beta \cos \theta}
$$

Where D is the average crystallite domain size perpendicular to the reflecting planes, $\lambda$ is the $X$ ray wave length, $\beta$ is the full width at half maximum and $\theta$ is the diffraction angle.

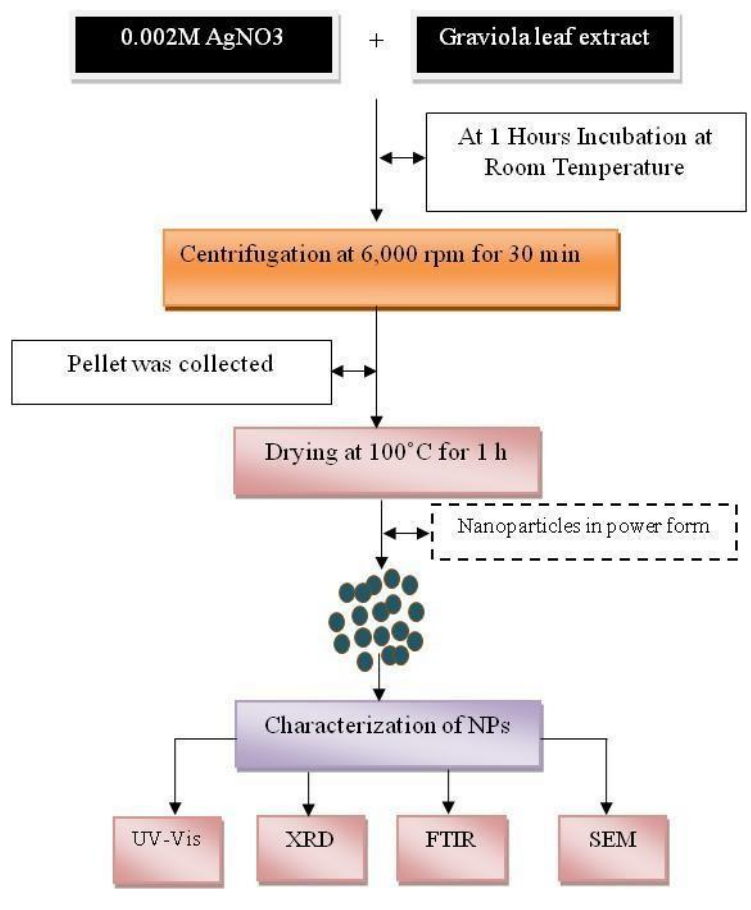

\subsubsection{Scanning Electron Microscopy}

Each of the colloidal solution containing silver nanoparticles was centrifuged at $6,000 \mathrm{rpm}$ for $30 \mathrm{~min}$. The supernatants were discarded and the final pellets were dissolved in $1000 \mu \mathrm{L}$ of deionized water. The pellet was mixed properly and carefully 
placed on a glass cover slip followed by air-drying. The cover slip itself was used during scanning electron microscopy (SEM) analysis. The images of silver nanoparticles were obtained in a scanning electron microscope (Fb-Quanta 200 SEM machine). The details regarding applied voltage, magnification used and size of the contents of the images were implanted on the images itself.

\section{RESULTS AND DISCUSSION}

Aqueous Silver nitrate solution was reducing during exposure to the Graviola leaf aqueous extract. The colour of the reaction mixture changed from pale yellow to reddish brown after 1 hour incubation at room temperature (Fig. 2) which indicates the formation of silver nanoparticles. It is well known that silver nanoparticles exhibit dark brown colour in water due to excitation of surface Plasmon vibration in metal nanoparticles. Control (without silver nitrate) shows no colour change with aqueous silver nitrate solution when incubated at same condition Control showed pale yellow colour solution with culture filtrate and Silver nanoparticles showed Dark brown colour solution after 1 hours of inhibitions. Shanmugavadivu et al. (2014) reported that the green synthesis of silver nanoparticles using pomegranate fruit peel extract to the aqueous solution of the silver nitrate the colour of the reaction medium changed rapidly from coloreless to brown.

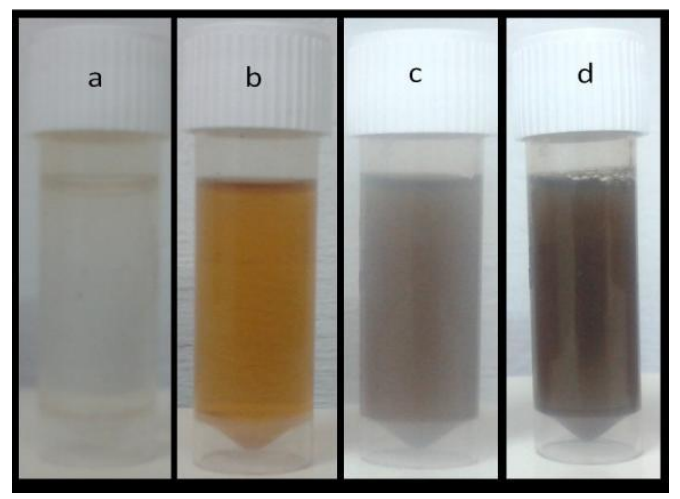

Fig. 2. Visual appearances of vials containing the aqueous extract of Graviola leaf and $\mathrm{AgNO}_{3}$ solution after $1 \mathrm{~h}$ reaction time

a) $0.002 \mathrm{M} \mathrm{AgNO}_{3}$ b) aqueous extract of Graviola leaf c) reaction mixture 5 min incubation d) reaction mixture after $1 \mathrm{~h}$ incubation at room temperature. The change of colour is an indication of the growth of silver nanoparticles.

\subsection{UV-Visible spectroscopy analysis}

Formation of silver nanoparticles (AgNPs) by reduction with $\mathrm{AgNO}_{3}$ (Silver nitrate) by aqueous extract of Graviola leaf samples were characterized by UV-Visible spectroscopy at $5 \mathrm{~min}$ interval time (JASCO-V/670) and this technique has proved to be very useful for the analysis of silver nanoparticles formation. In the VU-Vis absorption spectrum, a strong, broad peak located between 440 to $460 \mathrm{~nm}$ was observed (Fig. 3). UV-Visible spectra also revealed that formation of AgNPs occurred rapidly within the first $10 \mathrm{mins}$ only and the AgNPs in solution remained stable even after 1 hour of completion of reaction. Metal nanoparticles such as gold and silver have free electrons, which give rise to surface plasmon resonance absorption band. Biogenic synthesis areca nut aqueous extract medicated silver nanoparticles showed the surface plasmon resonance peak observed at $414 \mathrm{~nm}$ (Ranjithkumar et al., 2013).

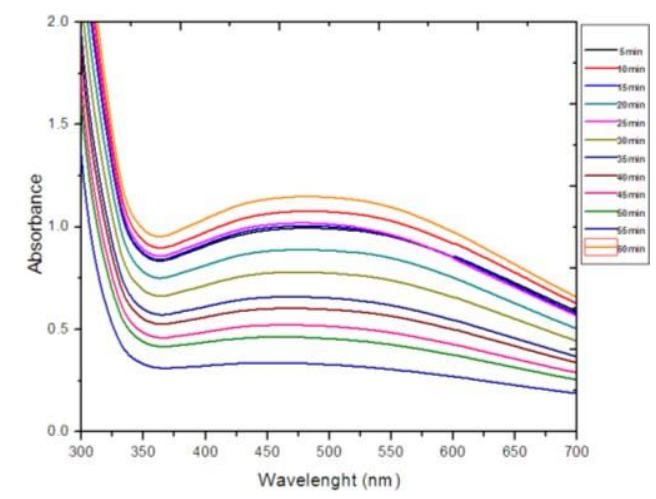

Fig. 3. Absorption spectrum of silver nanoparticles synthesized by aqueous extract of Graviola leaf

\subsection{Fourier transform infrared spectrum (FTIR) analysis}

The presence of some functional groups as revealed by FTIR spectral analysis is shown in Fig. 4. The FTIR spectral analysis of aqueous extract of Graviola leaf reduced silver nanoparticles showed shape absorbance peak at 1219.01, 1365.60, 1635.64 and $3371.57 \mathrm{~cm}^{-1}$ assigned to $\mathrm{C}-0$ stretching vibration of alcohols, carboxylic acids, esters, ether, alkanes and $\mathrm{N}-\mathrm{H}$ bending vibration of primary and secondary amines or amides. On other hand, synthesized silver nanoparticles showed light absorbance peak at 2152.56, 2183.42 and 2646.34 $\mathrm{cm}^{-1}$ assigned to $-\mathrm{C} \equiv \mathrm{C}$ - stretching vibration of alkynes, $\mathrm{C}-\mathrm{H}$ stretching vibration of aldehydes and $\mathrm{C} \equiv \mathrm{N}$ stretching vibration of nitriles. The amine and alkynes groups present in the sample this may be responsible for the reduction and capping of silver nanoparticles Vanaja et al. (2014) reported that the functional biomolecules are hydroxyl, carboxylic, phenol, and amine group in $M$. tinctoria leaf extract 
involved in the reduction of silver ions which was confirmed by FTIR spectrum. Moreover, in the present study indicated, the FTIR spectrum revealed various functional groups present at different position.

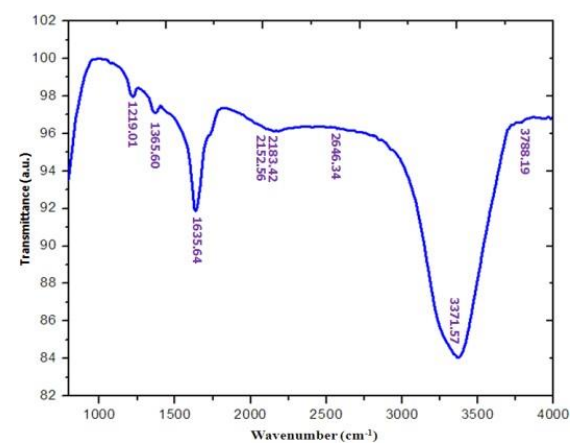

Fig. 4. FTIR spectrum of silver nanoparticles synthesized by aqueous extract of Graviola leaf.

\subsection{X-ray Diffraction spectrum (XRD) analysis}

Fig. 5 shows the X-ray diffraction spectrum of silver nano particles synthesized from aqueous extract of Graviola leaf at room temperature. The Braggs reflections were observed in the XRD pattern at $2 \theta=27.32,32.4$ and 46.4 These Braggs reflections clearly indicated the presence of (111), (200) and (402) sets of lattice planes and further on the basis that they can be indexed as face-centered-cubic (FCC) structure of silver. Hence XRD pattern thus clearly illustrated that the silver nanoparticles formed in this present synthesis are crystalline in nature and the average size of nanoparticles around 30-70 nm. Additional as yet unassigned peaks were also observed and suggesting that the crystallization of bioorganic phase occurred on the surface of the nanoparticles. The noise due to the protein shell surrounding the nanoparticles is visible from the spectrum.

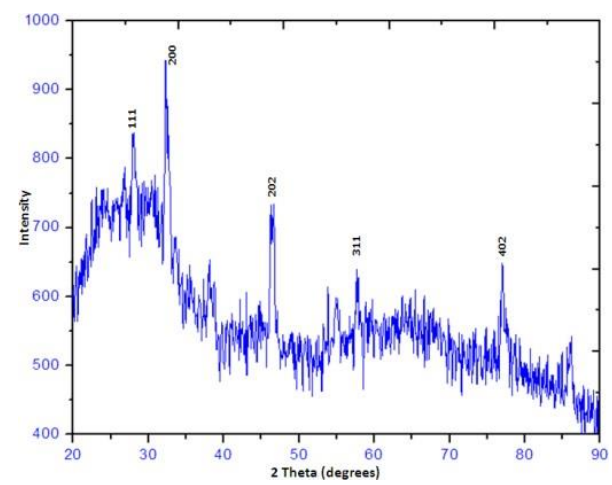

Fig. 5 X-ray diffraction spectrum of silver nanoparticles synthesized by aqueous extract of Graviola leaf.

\subsection{Scanning electron microscope (SEM) analysis}

The morphology of silver nanoparticles was determined by scanning electron microscope. SEM image of Graviola leaf medicated biosynthesized silver nanoparticles shows uniformly distributed silver nanoparticles on the surface of the cell. The green synthesized silver nanoparticles were spherical in shape with high aggregation. The larger silver nanoparticles may be due to the aggregation of the smaller ones. This may be due to availability of different quantity and nature of capping agents present in the aqueous extract of Graviola leaf. Ranjithkumar et al. (2013) reported areca nut medicated green synthesis silver nanoparticles showed spherical in shape with high agglomeration.

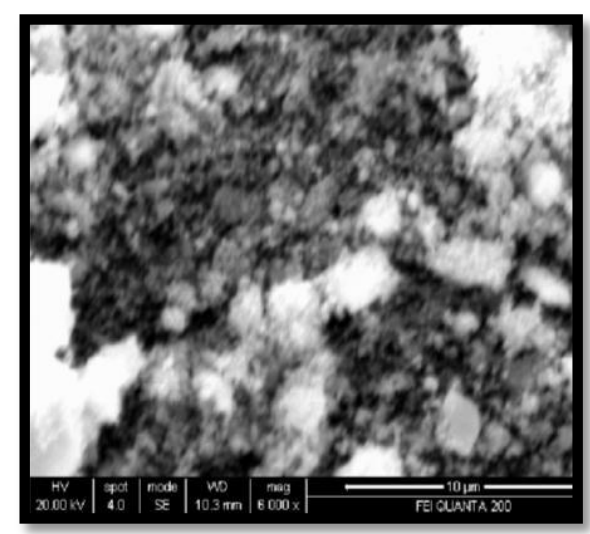

Fig. 6. SEM image of green synthesis silver nanoparticles using aqueous extract of Graviola leaf at room temperature.

\section{CONCLUSION}

A simple and eco-friendly green route was used to synthesis silver nanoparticles from silver nitrate using Graviola leaf aqueous extract. Graviola leaf extract is found suitable for the green synthesis of silver nanoparticles at $1 \mathrm{~h}$ incubation in room temperature. Colour change occur due to surface plasmon resonance during the reaction with the ingredients present in the Graviola leaf extract resulting in the formation of silver nanoparticles, which is confirmed by UV-vis spectroscopy, FT-IR, XRD and SEM. XRD and SEM analysis revealed spherical shaped nanoparticles of size about 30-70 nm.

\section{REFERENCES}

Protima, R., Siim Kuunal, Stanislav Ferdov, and Erwan Rauwel, (2015). A review on the green synthesis of silver nanoparticles and their morphologies studied via TEM. Adv. Mat. Sci. Eng. 1-9. 
Ranjithkumar, R., Selvam Kuppusamy, and M. Shanmugavadivu, (2013). Green synthesis of silver nanoparticles using areca nut extract for enhanced antibacterial activity. J. Green Sci. Tech., 1:102-106.

Ranjithkumar Rajamani, Selvam Kuppusamy and M. Shanmugavadivu, (2013). Antibacterial textile finishing via green synthesized silver nanoparticles. J. Green Sci. Tech., 1: 111-113.

Shanmugavadivu, M., Selvam Kuppusamy and R. Ranjithkumar, (2014). Synthesis of pomegranate peel extract mediated silver nanoparticles and its antibacterial activity. Am. J. Adv. Drug Del., 2(2): 174-182.

Vanaja, M., K. Paulkumar, M. Baburaja, S. Rajeshkumar, G. Gnanajobitha, C. Malarkodi G. Sivakavinesan, and G. Annadurai, (2014). Bioinorg. Chem. Appl. 8.

Yuliang Wang and Younan Xia, (2004). Bottom-Up and Top-Down Approaches to the Synthesis of Monodispersed Spherical Colloids of Low Melting-Point Metals. Nano Letters, 4(10): 2047-2050. 